\title{
Drug-Resistant Herpes Simplex Virus in HIV Infected Patients
}

\author{
Margarita S. Lolis, Lenis González, Philip J. Cohen, Robert A. Schwartz \\ Dermatology, New Jersey Medical School, Newark, New Jersey
}

\section{Corresponding author:}

Robert A. Schwartz, MD, MPH

Professor and Head, Dermatology

New Jersey Medical School

185 South Orange Street, Room H-576

Newark, NJ 07103

USA

roschwar@cal.berkeley.edu

Received: September 16, 2008

Accepted: October 2, 2008.

\begin{abstract}
SUMMARY Herpes simplex virus type 2 (HSV2) infection is a major source of morbidity in human immunodeficiency virus (HIV)-infected patients, since reactivations - whether symptomatic or asymptomatic - are associated with increased HIV viral load and viral shedding. Acyclovir, valacyclovir and famcyclovir are indicated for the treatment of HSV2 in HIV patients. This class of drugs has been shown to enhance survival in HIV-infected individuals. However, with the emergence of drug-resistant strains of HSV2, the rates of resistance among HIV patients are almost ten-fold those in immunocompetent individuals, comparing $0.6 \%$ to $6 \%$. These HSV2 infections tend to be more severe and to recur. More ominously, disease progression of HIV is promoted by concurrent infection with HSV2. Intravenous foscarnet and cidofovir may be used for acyclovir-resistant HSV; however, resistance to these drugs has been documented. Newer therapies such as the toll-like receptor agonist imiquimod and immunomodulating dipeptides offer promise for the treatment of HSV2 in HIV-infected individuals.
\end{abstract}

KEY WORDS: herpes simplex virus type 2, HIV infection, HSV2 therapy, HSV2 drug-resistance

\section{INTRODUCTION}

Herpes simplex virus (HSV) infection is a major cause of morbidity and even mortality in immunosuppressed individuals (1). Although both HSV1 and HSV2 infections occur more commonly among immunocompromised patients, HSV2 infection has been more extensively studied in the human immunodeficiency virus (HIV)-infected population. HSV2 infection has been identified as a risk factor for HIV acquisition and transmission. HSV2 may accelerate HIV progression, increasing HIV viral shedding and viral loads in plasma and genital secretions (2-4). Although HSV2 antiviral therapy has been shown to reduce HIV replica- tion, viral load and disease evolution, drug-resistant HSV2 strains have begun to emerge. This review focuses on the natural history of HSV2 infection in HIV-infected individuals, development of drug resistance in this population, and treatment options for drug-resistant HSV.

\section{HSV2/HIV SYNERGY}

\section{EPIDEMIOLOGY}

The synergistic interaction between HSV2 and HIV infections is supported by epidemiological data $(2,3,5-8,10,21)$. These studies demonstrate 
that HSV2 predisposes individuals to HIV acquisition and transmission, and accelerates HIV disease progression. HSV2 infection may be found in $50 \%-90 \%$ of HIV-patients but only $22 \%$ of immunocompromised patients (3-5). One study showed $\mathrm{HSV} 2$ to be associated with a two- to three-fold increase in the risk of HIV infection (5). A metaanalysis of 18 longitudinal studies found the HSV2 infection to be associated with an increased risk of HIV infection, with a relative risk of 3.1 in women, 2.7 in heterosexual men and 1.7 in homosexual men (7). Mathematical modeling has projected that the peak incidence of HIV may double in the next ten years due to the presence of HSV2 (2).

In co-infected individuals, acute HSV2 infections and reactivations may increase HIV replication and plasma viral load, with concomitantly increased genital HIV viral shedding and accelerated progression of HIV. Such HSV2 reactivations, more frequent and protracted in immunosuppressed individuals, may explain the greater transmissibility and faster disease progression in HIV-infected patients (6-8).

\section{PATHOGENESIS}

Several biological mechanisms may explain the synergy between HSV2 and HIV, which are described below.

\section{Disruption of mucosal integrity}

HSV2 infection causes genital ulcerations, clinically obvious or microscopic (9). Disruptions to the epidermal barrier, as well as asymptomatic viral shedding, may trigger an immune response, resulting in the activation of CD4+ cells as well as the recruitment of dendritic cells expressing dendritic cell-specific intercellular adhesion molecule3-grabbing nonintegrin (7). Dendritic cells and CD4+ cells are targets easily infected by HIV.

\section{HSV2-induced immune activation}

HSV2 infection increases HIV replication through immune mechanisms. For example, Tcell depletion by local and systemic immune activation may occur during acute HSV2 infection (8). HSV-mediated down-regulation of the cytokines interferon- $\gamma$ and interleukin-12 has been shown to increase HIV replication. Also, HSV2 activation of host toll-like receptors has been linked to HIV progression (7). These various mechanisms all lead to more rapid disease progression by increasing HIV replication and depleting T cells, which further weakens the immune response.

\section{HSV2 protein transactivation}

ICP0, ICP27, ICP4, IE 110, and IE175 are immediate-early HSV proteins, shown to increase HIV transcription in vitro (2). The interaction of ICP0, ICP27 and ICP4 with the HIV long terminal repeat (LTR) region induces the cellular activators nuclear factor kB (NF-kB) and Sp1. These activators ultimately up-regulate HIV replication (8). Also, these HSV proteins amplify HIV expression through post-transcriptional stabilization of HIV RNAs (2).

\section{Disruption of vaginal flora}

Bacterial vaginosis may play an intermediate role in the synergistic relationship of HIV and HSV2. HSV2 infection facilitates bacterial vaginosis. Normally, the lactic acid and hydrogen peroxide produced by vaginal lactobacilli maintain a low $\mathrm{pH}$, which kills pathogens. Bacterial vaginosis causes a loss of the lactobacilli; the resulting loss of lactic acid and hydrogen peroxide increases vaginal $\mathrm{pH}$, enabling the survival of HIV. Other abnormal bacterial flora of the vagina, such as Gardenella vaginalis, may recruit leukocytes and increase pro-inflammatory cytokines, which, in turn, may activate the HIV LTR region and ultimately increase HIV replication (8).

\section{CLINICAL MANIFESTATIONS OF HIV AND HSV2 CO-INFECTION}

Immunosuppressed patients experience frequent recurrences of HSV2 (1). These infections may be clinically atypical in appearance and location; they may be extensive, deeply ulcerated or necrotic. Due to low CD4+ cell counts, recurrences tend to be protracted and poorly responsive to therapy. The lesions may involve multiple sites and may have a longer duration of viral shedding (1).

Occasionally, HSV2 causes confluent verrucous papules and/or nodules on the penis, scrotum, vulva, intergluteal cleft, and other skin and mucosal areas, mimicking condyloma acuminatum, verrucous carcinoma, and other ulcerative diseases such as syphilis and chancroid $(1,5)$. Rarely, esophagitis, encephalitis, hepatitis, pneumonitis or retinal necrosis may occur in systemic and disseminated disease $(2,6)$.

\section{Asymptomatic HSV2 reactivation}

Most HSV2 reactivations are asymptomatic, with silent HIV viral shedding from mucosal sites. Such asymptomatic viral shedding correlates 
inversely to the CD4+ cell counts and may occur in greater quantity and at a greater number of anatomic sites in patients with lower CD4+ cell counts $\left(<400 \mathrm{cell} / \mathrm{mm}^{3}\right)$ (2). The rate of HSV2 shedding is highly correlated with plasma HIV RNA levels, and hence with more rapid disease progression (5). Highly active antiretroviral therapy (HAART) does not appear to alter the frequency of shedding or the titer of HSV2 shedding (3).

\section{ANTI-VIRAL HSV2 THERAPY AND HIV}

Since its emergence in 1983, acyclovir has been the mainstay of therapy for HSV infections. Acyclovir is a nucleoside analog, and its mechanism of action involves its phosphorylation by the HSV thymidine kinase (TK) to acyclovir monophosphate. Cellular kinases further phosphorylate acyclovir monophosphate to acyclovir triphosphate $(1,2,15)$. Since viral DNA polymerase has a higher affinity for acyclovir triphosphate than human cellular DNA polymerase, it may be incorporated into the growing viral DNA chain, resulting in chain termination and inhibition of viral DNA replication (2).

Numerous studies have indicated that HSV antiviral therapy enhances survival in HIV patients. Three randomized controlled trials have shown that treatment with oral valacyclovir decreases genital and plasma HIV replication (11-13). In another randomized trial, a greater than $0.5 \log _{10}$ copies/mL reduction in HIV blood viral load was observed after oral acyclovir therapy (14). A metaanalysis of 8 randomized trials indicated that oral acyclovir offered a significant survival benefit for HIV-infected persons (hazard ratio, 0.78; $95 \% \mathrm{Cl}$, 0.65-0.93) (5).

The exact mechanism by which antiviral HSV therapy enhances survival in HIV patients is unknown but various mechanisms have been proposed. Studies have suggested that HSV suppression may interfere with HIV up-regulation and may reduce HIV shedding in herpetic lesions within 4-5 days of therapy initiation (5). Women treated with valacyclovir suppressive therapy had a mean of $0.35 \mathrm{log}$ copies/mL cervicovaginal HIV levels lower than untreated females (11). Furthermore, a reduction in the recurrence of HSV ulcers and viral shedding may produce an overall beneficial effect on HIV progression (7).

\section{DRUG RESISTANCE IN HIV PATIENTS}

Acyclovir resistance was first documented shortly after its emergence. In immunocompetent individuals, the prevalence of resistance is as high as $0.6 \%$. By contrast, in immunocompromised individuals, the prevalence is almost ten times higher, ranging between $3 \%$ and $6 \%$ (15). A recent study indicated that $10 \%$ of HSV isolates obtained from AIDS-infected patients were resistant to acyclovir (16). Resistance to other classes of antivirals such as foscarnet has been documented as well. In a study of patients with excreted acyclovir-resistant HSV strains, $61 \%$ of those treated with foscarnet subsequently developed resistance to this drug (15).

The generation of drug-resistant HSV strains may be attributed to the prolonged use of antiviral medications in immunocompromised patients. The extended use of antiviral medications may lead to the selection of nucleoside analog-resistant HIV strains resistant in 7\%-14\% of immunocompromised hosts (17). This can occur quickly, as resistant strains have been isolated as early as 17 hours after initiation of pencyclovir and two days after initiation with acyclovir (15).

Host-related factors include the strength of the immune response mounted against HSV infection. In an immunocompromised individual, an attenuated or negligible immune response against HSV2 infection may result in the continuous replication of both wild type and resistant strains. The prolonged use of antiviral medications may further select mutant strains (18).

Drug-resistant HSV strains usually originate from mutations in thymidine kinase (TK) and DNA polymerase. Three types of acyclovir-resistant strains have been identified. These include 1) TK negative mutants that result from a deletion of the TK gene; 2) TK partial mutants that have reduced TK activity due to a mutation in the gene; and 3) TK altered mutants that phosphorylate thymidine but not acyclovir. Approximately $95 \%$ of acyclovir-resistant mutants lack TK activity or have reduced levels of the enzyme. Strains with mutations in DNA polymerase and helicase-primase inhibitors are much less common $(18,19)$. These mutations frequently arise spontaneously. Notably, in vitro acyclovir resistance may not correlate with clinical resistance (2).

\section{THERAPY}

Acyclovir, valacyclovir and famcyclovir are indicated for the treatment and suppression of both primary and recurrent HSV2 infections. Acyclovirresistant HSV2 isolates are usually not responsive to treatment with valacyclovir or famcyclovir due to 
mutations in TK. In this case, therapy with a non TK-dependent drug, such as intravenous foscarnet which targets viral DNA polymerase, may be initiated. Resistance to foscarnet on the basis of viral mutation has been documented. Intravenous cidofovir has been reported clinically effective for acyclovir- and foscarnet-resistant HSV2 strains (20).

Imiquimod is a toll-like receptor (TLR) 7 agonist, which targets both innate and cell-mediated pathways of the immune system. Imiquimod promotes Th1 cell-mediated immune response (22), inhibits Th2 response (23), and induces functional maturation of Langerhans cells. This drug induces lymphocytes to produce numerous cytokines such as interferon- $\alpha$, known to have direct antiviral activity (21). This increased activation of the innate and cytotoxic-specific immune response is particularly helpful for immunocompromised patients (21). Resiquimod, an investigational analog of imiquimod with mixed TLR 7/8 activity, has had mixed success (22).

Clinical reports on treatment of HSV-resistant infections with imiquimod describe rapid improvement of skin lesions. In guinea pigs, topical imiquimod is associated with a decrease in the number of primary lesions of HSV2 infections, a decrease in the number of episodes of viral shedding, and a reduction in viral load measured in the cerebrospinal fluid (23).

Helicase-primase inhibitors (HPIs) target the HSV helicase primase complex. This class of drugs is potent with low rates of resistance. It appears superior to nucleoside analogs in animal models. The use of HPIs in synergy with nucleoside analogs may delay the emergence of drugresistant strains $(19,24)$.

Distamycin A, a basic antibiotic belonging to the category of lexitropsin DNA minor groove binding drugs, has significant antiviral action against HSV2. Distamycin A inhibits activity of all DNA polymerases by forming a stable complex with double-stranded DNA (25).

The immunomodulating dipeptide SCV-07 is another promising therapy for HSV2 infections. Guinea pigs infected genitally with HSV2 had significantly reduced frequency and severity of recurrent disease when treated with SCV-07. Further investigation is needed (26).

\section{CONCLUSION}

The relationship between HSV2 infection and HIV is complex. HSV2 infection promotes HIV acquisition, transmission and disease progression by increasing viral loads and episodes of viral shedding. On the other hand, HIV infection increases the duration, severity and frequency of episodes of HSV2 reactivations. Although the exact mechanisms of these synergistic interactions are unknown, numerous explanations have been proposed. Treatment of HSV2 infections with antiviral agents enhances survival for HIV patients by decreasing HIV replication, lowering viral loads, and reducing the number of episodes of viral shedding.

It appears reasonable that HIV-positive individuals be evaluated for concurrent HSV2 infection and possibly be offered long-term suppressive treatment. HSV2 resistance testing may be useful in patients with demonstrated clinical failure of HSV2 therapy to oral acyclovir, valacyclovir, or famcyclovir. Although intravenous foscarnet and cidofovir have been used successfully for resistant strains, renal toxicity is a serious side effect of these drugs and therefore should be used with caution. Topical imiquimod may be of particular value, as it is clinically effective against resistance with HSV2 infection and has not been associated with any serious side effects.

\section{References}

1. Fatahzadeh M, Schwartz RA. Human herpes simplex virus infections: epidemiology, pathogenesis, symptomatology, diagnosis, and management. J Am Acad Dermatol 2007;57:73763.

2. Lingappa JR, Celum C. Clinical and therapeutic issues for herpes simplex virus 2 and HIV co-infection. Drugs 2007;67:155-74.

3. Corey L. Synergistic copathogens-HIV-1 and HSV-2. N Engl J Med 2007;356: 854-6.

4. Cernik C, Gallina K, Brodell RT. The treatment of herpes simplex infections. Arch Intern Med 2008;168:1137-44.

5. Strick LB, Wald A, Celum C. Management of herpes simplex virus type 2 infection in HIV type 1 infected persons. Clin Infect Dis 2006;43:347-56.

6. Celum C, Wald A, Hughes J, Sanchez J, Reid $\mathrm{S}$, Delany-Moretlwes S, et al. Effect of acyclovir on HIV-1 acquisition in herpes simplex virus 2 seropositive women and men who have sex with men: a randomized, double blind, placebo-controlled trial. Lancet 2008;371:2109-19.

7. Sheth PM, Sunderji S, Shin LYY, Rebbapragada A, Huiber S, Kimani J, et al. Coinfection 
with herpes simplex virus type 2 is associated with specific $T$ cell responses and immune activation. J Infect Dis 2008;197:1394-401.

8. Van de Perre $P$, Segondy M, Foulogne V, Ouedraogo A, Konate I, Huraux JM, et al. Herpes simplex virus and HIV-1: deciphering viral synergy. Lancet Infect Dis 2008;8:490-7.

9. Wald A, Corey L. How does herpes simplex virus type 2 influence human immunodeficiency virus infection and pathogenesis? J Infect Dis 2007;195:1509-12.

10. Corey L. Herpes simplex virus type 2 and HIV1: the dialogue between the 2 organisms continues. J Infect Dis 2007;195:1242-4.

11. Nagot N, Ouedraogo A, Foulongne V, Konate I, Weiss HA, Vergne L, et al. Reduction of HIVRNA levels with therapy to suppress herpes simplex virus. N Engl J Med 2007;356:790-9.

12. Ouedraogo A, Nagot N, Vergne L, Konate I, Weiss HA, Defer MC, et al. Impact of suppressive herpes therapy on genital HIV-RNA among women taking antiretroviral therapy: a randomized controlled trial. AIDS 2006;20:230513.

13. Zuckerman R, Lucchetti A, Whittington W, Sanchez J, Coombs RW, Zuniga R, et al. HSV suppression with valacyclovir reduces rectal and blood plasma HIV levels in HIV, HSV2 seropositive men: a randomized, double blind placebo controlled crossover trial. J Infect Dis 2007;196:1500-8.

14. Cooper DA, Pehrson PO, Pedersen C, Moroni M, Oksenhendler E, Rozenbaum W, et al. The efficacy and safety of zidovudine alone or as cotherapy with acyclovir for the treatment of patients with AIDS and AIDS-related complex: a double-blind randomized trial. AIDS 1993;7:197-207.

15. Danve-Szatanek C, Aymard M, Thouvenot D, Morfin F, Agius G, Bertin I, et al. Surveillance network for herpes simplex virus resistance to antiviral drugs; 3-year follow-up. J Clin Microbiol 2004;42:242-9.

16. Ziyaeyan M, Alborzi A, Japoni A, Kadivar M, Davarpanah MA, Pourabbas B, et al. Frequency of acyclovir-resistant herpes simplex viruses isolated from the general immunocom- petent population and patients with acquired immunodeficiency syndrome. Int $\mathrm{J}$ Dermatol 2007;46:1263-6.

17. Weinberg A, Leary JJ, Sarisky RT, Levin MJ. Factors that affect in vitro measurement of the susceptibility of herpes simplex virus to nucleoside analogues. J Clin Virol 2007;38:139-45.

18. Levin MJ, Bacon TH, Leary JJ. Resistance of herpes simplex virus infections to nucleoside analogues in HIV-infected patients. Clin Infect Dis 2004;39:S248-57.

19. Biswas S, Field HJ. Herpes simplex virus helicase-primase inhibitors: recent findings from the study of drug resistance mutations. Antivir Chem Chemother 2008;19:1-6.

20. Saint-Leger E, Fillet AM, Malvy D, Rabanel B, Caumes E. Efficacy of cidofovir in an HIV infected patient with an acyclovir and foscarnet resistant herpes simplex virus. Ann Dermatol Venereol 2001;128:747-9.

21. Martinez V, Molina JM, Scieux C, Ribaud $P$, Morfin F. Topical imiquimod for recurrent acyclovir-resistant HSV infection. Am J Med 2005;119:e9-11.

22. Miller RL, Meng TC, Tomai MA. The antiviral activity of toll-like receptor 7 and $7 / 8$ agonists. Drug New Prospect 2008;21:61-87.

23. Gilbert J, Drehs MM, Weinberg JM. Topical imiquimod for acyclovir-unresponsive herpes simplex virus 2 infection. Arch Dermatol 2001;137:1015-7.

24. Biswas S, Tiley LS, Zimmermann H, Birkmann A, Field HJ. Mutations close to functional motif IV in HSV01 UL5 helicase that confer resistance to HSV helicase-primase inhibitors, variously affect viral growth rate and pathogencitiy. Antiviral Res 2008. 2008;80:81-5.

25. Matteoli B, Bernardini S, luliano R, Parenti S, Freer $\mathrm{G}$, Broccolo $\mathrm{F}$, et al. In vitro antiviral activity of distamycin A against clinical isolates of herpes simplex virus 1 and 2 from transplanted patients. Intervirology 2008;51:166-72.

26. Rose WA, Tuthill C, Pyles RB. An immunomodulating dipeptide, SCV-07, is a potential therapeutic for recurrent genital herpes simplex virus type 2 (HSV-2). Int J Antimicrob Agents 2008. 2008;32:262-6. 